\title{
The accumulation of macrophages attenuates the effect of recombinant human endostatin on lung cancer
}

This article was published in the following Dove Press journal:

OncoTargets and Therapy

25 October 2016

Number of times this article has been viewed

\author{
Yanyang Liu ${ }^{1, *}$ \\ Li Tu',* \\ Li Wangl,* \\ Jianlin Long' \\ Jiantao Wang' \\ Yuyi Wang' \\ Feng Luo' \\ Dan $\mathrm{Cao}^{2}$
}

'Department of Medical Oncology, Lung Cancer Center, ${ }^{2}$ Department of Abdominal Oncology, Cancer Center and State Key Laboratory of Biotherapy, West China Hospital, Sichuan University, Chengdu, Sichuan, People's Republic of China

*These authors contributed equally to this work
Correspondence: Dan Cao

Department of Abdominal Oncology,

Cancer Center and State Key Laboratory

of Biotherapy, West China Hospital of

Sichuan University, 37 Guo Xue Xiang,

Chengdu 6I004I, Sichuan, People's

Republic of China

Email caodan3I6@I63.com

Feng Luo

Department of Medical Oncology, Lung Cancer Center, Cancer Center and State Key Laboratory of Biotherapy, West China Hospital, Sichuan University, 37 Guo Xue Xiang, Chengdu, Sichuan, 6I004I, People's Republic of China Email luofeng@medmail.com.cn
Background: Although anti-angiogenic therapy is widely applied clinically, its efficacy has been less than expected. Screening for regulatory factors and sensitive indicators to define the effectiveness of these drugs is required. Through a retrospective study of clinical data, we found that patients with a higher peripheral monocyte-to-lymphocyte ratio (MLR) obtained less benefit from recombinant human endostatin (rhES, Endostar $\left.{ }^{\circledR}\right)$, an anti-angiogenic drug, in lung cancer. Because MLR is positively correlated with macrophage count in tumors, this result suggests that macrophages may influence the effectiveness of rhES therapy in lung cancer.

Methods: Clinical data from 72 lung cancer patients treated with rhES were collected. Animal study, flow cytometry, immunofluorescence, enzyme-linked immunosorbent assay, Western blot analysis, and transwell migration assays were carried on Lewis lung carcinoma (LLC) cells, bone marrow-derived macrophages, macrophage cell line RAW264.7, and ANA-1 cells.

Results: Clinical data showed that compared with the baseline MLR before rhES treatment, patients with progressive disease had higher MLRs than those of patients with partial response. Experimental results showed that more macrophages were recruited in the LLC tumors after rhES treatment and the majority of them displayed an M2-like phenotype. rhES aggravated hypoxia and the inflammatory response in the tumor microenvironment. Hypoxia promoted the expression of CCL2 by endothelial and fibroblast cells, which could induce macrophages recruitment, and increased levels of inflammatory cytokines (interleukin-4 [IL-4], IL-6, and IL-10) skewed macrophage polarization toward the M2-like phenotype. Hypoxia or inflammation cytokine-treated macrophages enhanced the progression of LLC in vitro and in vivo.

Conclusion: We found rhES could aggravate hypoxia and the inflammatory response in the tumor microenvironment. These changes were favorable for macrophage accumulation, and skewed their polarization toward the M2-like phenotype which could help LLC to escape from the anti-angiogenic therapy. Thus, these data indicate the accumulation of macrophages in the tumor microenvironment may adversely affect the efficacy of rhES on lung cancer.

Keywords: macrophage, anti-angiogenic therapy, recombinant human endostatin, lung cancer, tumor microenvironment

\section{Introduction}

Malignant tumors have become one of the most common causes of death in the world. Among these tumors, lung cancer is the leading cause of cancer incidence and mortality. With the low rate of early diagnosis and high-grade malignancy in lung cancer, the 5 -year survival rate of this disease is only about $20 \% .{ }^{1,2}$ Thus, more effective treatments are required. 
Recently, anti-angiogenic therapy has shown a survival advantage in many cancers. ${ }^{3}$ Recombinant human endostatin (rhES, Endostar ${ }^{\circledR}$; Simcere-Medgenn Bio-Pharmaceutical Co., Ltd., Nanjing, People's Republic of China), an antiangiogenic drug, has anticancer effects in a variety of tumors, and is now used to treat lung cancer in People's Republic of China. However, clinical evidence also shows that antiangiogenic drugs are not as efficacious as expected and many people achieve only a short increase in survival time. ${ }^{4}$ From our clinical practice and other studies, ${ }^{5}$ it is apparent that not all lung cancer patients can benefit from rhES. Thus, it is necessary to identify the regulatory factors and sensitive indicators of these agents so that the therapy can be optimized.

Previous studies have reported that the inflammatory status plays an important role in resistance to anti-angiogenic therapy. ${ }^{6}$ Patients receiving bevacizumab with higher peripheral mononuclear counts benefit less in terms of survival than patients with lower counts. ${ }^{7}$ Through a retrospective analysis of clinical data, we also found that lung cancer patients with a high peripheral monocyte-to-lymphocyte ratio (MLR) received little benefit from rhES treatment. These data suggest the peripheral monocyte count has a correlation with the clinical outcome of anti-angiogenic drug, but the mechanism of this phenomenon is unknown. According to existing evidence, the peripheral monocyte count correlate with poor prognosis in many cancers, because these cells are important sources of macrophages and myeloid-derived suppressor cells (MDSCs) in the tumor microenvironment. ${ }^{8}$ Macrophages and MDSCs are well known for their pleiotropic roles in mediating cancer progression and metastasis..$^{9,10}$ Our previous study found that MDSCs were associated with tumor refractoriness to rhES. ${ }^{11}$ Thus, we hypothesized that macrophages in the tumor microenvironment may also affect the efficacy of rhES.

To validate our hypothesis, we determined the number and polarization changes displayed by macrophages within the tumor during rhES treatment, and analyzed the impact of these cells on rhES therapy.

\section{Materials and methods Clinical data analysis}

We retrospectively reviewed 72 patients who visited the West China Hospital between 2007 and 2015. Data were extracted from patient medical records. Data with regard to baseline characteristics, including age, gender, performance status, stage, and treatment history, were collected. All patients were confirmed to have non-small cell lung cancer (NSCLC) with pathologic diagnosis. Patients had received two cycles of chemotherapy plus rhES $\left(7.5 \mathrm{mg} / \mathrm{m}^{2} /\right.$ day, 14 days as a cycle; Simcere-Medgenn Bio-Pharmaceutical Co., Ltd.). Tumor responses were analyzed according to the Response Evaluation Criteria in Solid Tumors (RECIST) version 1.1. ${ }^{8}$ According to the tumor responses, patients were divided into three groups: partial response (PR), stable disease (SD), and progressive disease (PD). Pretreatment complete blood count (CBC) profiles at the first-cycle therapy and the second-cycle therapy were determined. The MLR was calculated by dividing the absolute monocyte count by the absolute lymphocyte count., ${ }^{7,8}$ This study was approved by the Ethical Committee of West China Hospital and written informed consent was obtained from the participants for using their clinical records in this study.

\section{Cell lines and culture}

Lewis lung carcinoma (LLC) cells, macrophage cell lines RAW264.7 and ANA-1, human umbilical vein endothelial cells (HUVECs), and human skin fibroblasts (HSFs) were obtained from the Sichuan university (Chengdu, People's Republic of China), and maintained in Dulbecco's Modified Eagle's Medium (DMEM; Thermo Fisher Scientific, Waltham, MA, USA) containing 10\% fetal bovine serum (FBS) in a humid chamber at $37^{\circ} \mathrm{C}$ with $5 \% \mathrm{CO}_{2}$.

\section{Preparation of bone marrow-derived macrophages (BMDMs)}

Bone marrow isolation was performed as described previously. ${ }^{12}$ The bone marrow cells were differentiated into BMDMs with macrophage colony-stimulating factor (M-CSF) (Peprotech, Rocky Hill, NJ, USA). After 3 days of incubation, BMDMs were rinsed with DMEM to remove nonadherent cells and then cultured with $25 \mathrm{ng} / \mathrm{mL}$ interleukin-4 (IL-4), or $25 \mathrm{ng} / \mathrm{mL}$ IL-6, or $25 \mathrm{ng} / \mathrm{mL}$ IL-10 for additional 96 hours.

\section{Conditioned media (CM) preparation}

BMDMs were cultured with $25 \mathrm{ng} / \mathrm{mL}$ IL-4, or $25 \mathrm{ng} / \mathrm{mL}$ IL-6, or $25 \mathrm{ng} / \mathrm{mL}$ IL-10 (all Peprotech) for 96 hours. Then macrophages were incubated in serum-free media for 24 hours, after which culture supernatants were collected as CM. ${ }^{13}$ BMDMs were incubated in serum-free medium and cultured in a hypoxia incubator filled with a mixture of $1 \% \mathrm{O}_{2}, 5 \% \mathrm{CO}_{2}$, and $94 \% \mathrm{~N}_{2}$ for 24 hours. Then the culture supernatants were collected as hypoxia-conditioned media (Hypoxia-CM).

\section{Animal tumor models and treatment}

C57BL/6 mice (male, 5-6 weeks old; Beijing HFK Bioscience Co., Ltd, Beijing, People's Republic of China) 
were maintained in the Laboratory for Animal Experiments of Sichuan University under specified pathogen-free conditions with animal food and water. Mice were injected subcutaneously into the right axillary fossa with LLC cells $\left(1 \times 10^{6} / 0.1 \mathrm{~mL}\right)$. The tumor volume $\left(\mathrm{mm}^{3}\right)$ was calculated as length $\times$ width $^{2} / 2$. When the tumors reached volumes of about $100 \mathrm{~mm}^{3}$, mice were randomly assigned to treatment and control groups with seven mice in each group. For the treatment (rhES) group, $20 \mathrm{mg} / \mathrm{kg}$ rhES (Simcere-Medgenn Bio-Pharmaceutical Co., Ltd.) was injected intravenously (iv, by caudal vein injection) for 14 days, and the control group was given the same amount of normal saline (NS group). On days 7 and 14 after initiation of treatment, mice (three from each group) were sacrificed to harvest tumor tissues for subsequent analyses. ${ }^{14}$ Experimental procedures and protocols were approved by the Animal Ethics Committee of Sichuan University.

\section{Flow cytometry}

LLC tumors were washed with ice-cold phosphate-buffered saline (PBS, pH 7.4), and then dissociated by mincing the tissue with scalpels, followed by addition of DMEM media containing $1 \mathrm{mg} / \mathrm{mL}$ collagenase I and incubation for 120 minutes at $37^{\circ} \mathrm{C}$. The dissociated tumor tissue was then washed with ice-cold PBS and filtered through a $70 \mu \mathrm{m}$ cell strainer (BD Biosciences, Franklin Lakes, NJ, USA). The cell suspension was then centrifuged at a speed of 1,500 rpm for 5 minutes at $4^{\circ} \mathrm{C}$. The cells were resuspended in PBS for further analysis. Then the cell suspensions were stained with fluorochrome-labeled antibody-targeting murine CD11b-PECY7, F4/80-PE, CD197-APC (all eBioscience, San Diego, CA, USA), CD206-APC (Biolegend, San Diego, CA, USA), or an appropriate isotype control antibody and were analyzed by flow cytometry (Beckman Coulter, Brea, CA, USA). Results were analyzed using Kaluza Analysis Software version 1.3 (Beckman Coulter).

BMDMs, RAW 264.7, and ANA-1 cells were cultured with $25 \mathrm{ng} / \mathrm{mL}$ IL-4, or $25 \mathrm{ng} / \mathrm{mL}$ IL-6, or $25 \mathrm{ng} / \mathrm{mL}$ IL-10 (Peprotech) for 96 hours. Then the cells were stained with fluorochrome-labeled antibody-targeting murine F4/80-PE (eBioscience), CD206-APC (Biolegend), CD86-APC-CY7 (Biolegend) and were analyzed by flow cytometry.

LLC cells were cultured with indicated CM for 48 hours, and then cells were stained with an ALDEFLUOR ${ }^{\mathrm{TM}}$ kit (StemCell Technologies, Vancouver, CA, USA) according to the manufacturer's protocol. ${ }^{15}$ Then flow cytometry was used to analyze the population of cells with positive aldehyde dehydrogenase (ALDH) enzymatic activity.

\section{Histology, immunohistochemistry, and immunofluorescence}

LLC tumors were rapidly frozen at $-80^{\circ} \mathrm{C}$ or fixed with formalin and embedded in paraffin. Paraffin (thickness $5 \mu \mathrm{m}$ ) sections were routinely stained with hematoxylin and eosin (H\&E) for pathologic histology examination to evaluate the necrosis cells in the tumors. Paraffin sections were stained with DeadEnd ${ }^{\mathrm{TM}}$ Fluorometric TUNEL Kit (Promega Corporation, Madison, WI, USA) for pathologic histology examination to evaluate the apoptosis cells in the tumors.

The frozen sections (thickness $5 \mu \mathrm{m}$ ) were used to detect expression of F4/80 and CD31. Sections were incubated at $4^{\circ} \mathrm{C}$ overnight with one of the following primary antibodies: anti-mouse F4/80 or anti-mouse CD31 (both eBioscience). The sections were then incubated with appropriate secondary antibodies (ZSGB-BIO, Beijing, People's Republic of China) for detection by immunofluorescence or immunohistochemistry. Nuclei were highlighted using 4',6-diamidino-2-phenylindole (DAPI; Sino Biological Inc, Beijing, People's Republic of China) for 5 minutes. Sections were viewed and digitally photographed using a fluorescence microscope (Olympus UIS2, Tokyo, Japan). CD31 ${ }^{+}$cells were assessed in at least three random fields (three mice per group) at $\times 200$ magnification for statistical analysis.

\section{Detection of tumor hypoxia}

Hypoxyprobe ${ }^{\mathrm{TM}}$-1 Kit (HPI, Burlington, MA, USA) was used. ${ }^{16}$ In brief, mice were injected with $60 \mathrm{mg} / \mathrm{kg}$ of pimonidazole via the caudal vein and sacrificed 20 minutes later. Tumor tissues were harvested and rapidly frozen. The frozen sections (thickness $5 \mu \mathrm{m}$ ) were used for the observation of hypoxic regions. The Hypoxyprobe ${ }^{\mathrm{TM}}-1 \mathrm{Kit}$, consisting of $100 \mathrm{mg}$ solid pimonidazole $\mathrm{HCl}$ and $1 \mathrm{~mL}$ fluorescein isothiocyanate (FITC)-labeled mouse IgG1 monoclonal antibody against protein adducts of pimonidazole, was used to detect pimonidazole-protein adducts in tumor sections.

\section{Enzyme-linked immunosorbent assay (ELISA)}

LLC tumors were ground into powder with liquid nitrogen in grinding bowls, and then homogenized in radio immunoprecipitation assay (RIPA) lysis buffer (Beyotime, Shanghai, People's Republic of China), followed by centrifugation at 12,500 rpm for 15 minutes at $4^{\circ} \mathrm{C}$. BCA Protein Assay Kit (Beyotime) was used to test the protein concentration of samples. The prepared samples were stored at $-80^{\circ} \mathrm{C}$ until further analysis. Levels of vascular endothelial growth factor (VEGF), IL-2, IL-4, IL-6, and IL-10 in the samples were 
assessed by mouse ELISA kits (Neobioscience, Shenzhen, People's Republic of China) according to the manufacturer's protocol, and the colorimetric reaction was measured at $450 \mathrm{~nm}$ using a microplate reader (Benchmark Electronics, Angleton, TX, USA).

BMDMs and RAW264.7 cells were exposed or not exposed to IL-4 for 96 hours, and then cells were incubated in serum-free media for another 24 hours under hypoxic $\left(1 \% \mathrm{O}_{2}\right)$ or normal conditions. The culture supernatants were collected. The level of VEGF in the media was measured by ELISA kit (Neobioscience).

\section{Western blotting}

The samples of LLC tumors were collected as described earlier. Equal amounts of protein were separated by $8 \%-12 \%$ SDS-PAGE and transferred to polyvinylidene fluoride (PVDF) membranes (Merck Millipore, Billerica, MA, USA) by electroblotting. The membranes were probed with specific antibodies (CCL2; Zen-Bioscience, Chengdu, People's Republic of China). Blots were developed with horseradish peroxidase (HRP)-conjugated secondary antibodies and chemiluminescent substrate on Kodak X-ray film.

HUVECs, HSF, BMDMs, RAW264.7, and LLC cells were cultured as indicated. Then cells were harvested and homogenized in RIPA buffer. The protein levels of CCL2, MMP-9 (both Zen-Bioscience), E-cadherin, N-cadherin, vimentin (all Cell Signaling Technology, Danvers, MA, USA) were evaluated with Western blot analysis as described earlier.

\section{Reverse transcription-polymerase chain reaction (RT-PCR)}

The quantitative real-time RT-PCR analysis was performed by using TAKARA SYBR Premix EXTaqTM (TAKARA BIOTECHNOLOGY, Dalian, People's Republic of China). The reaction mixtures containing SYBR Green were composed following the manufacturer's protocol. The sequences of the primers used were as follows: ARG1: 5'-CACTCCCCTGACAACCAGCT-3' and 5'-AGGACACAGGTTGCCCATG-3'; YM1: 5' - TCTCTACTCCTCAGAACCGTCAGA-3' and 5' - GA T G T T T G T C C T T A G GA G G GC T T C - 3'; ACTIN: 5'-GGTCATCACTATTGGCAACG-3' and 5'-ACGGATGTCAACGTCACACT-3'.

\section{Migration assay}

Migration assay for myeloid monocytes, RAW264.7, ANA-1, and LLC cells was performed in a transwell Boyden chamber (BD Biosciences). Cell suspension $\left(3 \times 10^{5}\right.$ cells $\left./ \mathrm{mL}\right)$ was placed in the upper chamber. The lower compartment contained $0.6 \mathrm{~mL}$ of recombinant murine CCL2 (Peprotech) or indicated CM. After 24 hours of incubation at $37^{\circ} \mathrm{C}$, myeloid monocytes in the lower chamber were counted with Fuchs-Rosenthal counting chamber. RAW264.7, ANA-1, and LLC cells under the upper chamber were fixed with methanol and stained with $0.5 \%$ crystal violet (Beyotime) for 20 minutes. The stained cells were subsequently photographed and counted using a microscope (Olympus UIS2; $\times 200$ magnification, at least three random fields/well). ${ }^{14}$

\section{Wound healing assay}

LLC cells were seeded in six-well plates and cultured until $70 \%-80 \%$ confluent. An artificial wound was created by making a straight scratch with a pipette tip. Then the cells were treated with CM, and 24 hours later the migration of cells across this artificial wound was assessed.

\section{LLC metastasis model}

C57BL/6 mice (male, 5-6 weeks old; Beijing HFK Bioscience Co., Ltd) were used for experiments. All animal experiments were carried out in accordance with the Institutional Animal Use and Care Committee. BMDMs were exposed to either IL-4 for 96 hours or hypoxic condition $\left(1 \% \mathrm{O}_{2}\right)$ for 24 hours, and then the CM (+IL-4-CM and Hypoxia-CM) were obtained as described earlier. FBS was added to the CM to obtain a final concentration of $0.5 \%$. LLC cells were cultured with +IL-4-CM or Hypoxia-CM for 48 hours. Mice were randomly chosen and assigned to three groups (five animals per condition): control group, +IL4-CM group, and Hypoxia-CM group. Then LLC cells $\left(1 \times 10^{6} / 0.1 \mathrm{~mL}\right)$ were injected into mice iv from tail vein. Mice were sacrificed after 21 days, and the number of lung metastases nodes was determined grossly and microscopically. ${ }^{17}$

\section{Statistical analysis}

For quantitative data analysis, the results were plotted as the mean \pm standard deviation or median \pm interquartile range. Statistical analysis was performed using SPSS version 22.0 (IBM Software, New York, NY, USA). Statistical significance was determined using Student's $t$-test or Kruskal-Wallis test. $P<0.05$ was considered statistically significant.

\section{Results}

A total of 72 patients were included in the study. All patients received two cycles of chemotherapy plus rhES. CBC results before patients received treatment were used as baseline data. After two cycles of treatment, tumor responses were 
determined by imaging examination and were analyzed with RECIST version 1.1. Among the 72 patients, 21 achieved a PR, 36 exhibited SD, and 15 had PD (Figure 1A). In the PR group, the median \pm interquartile range of the peripheral MLRs before the first- and second-treatment cycles were $0.23 \pm 0.11$ and $0.21 \pm 0.15$, respectively. In the PD group, the MLRs were $0.41 \pm 0.19$ and $0.44 \pm 0.17$ before the first and second cycles of therapy, respectively (Figure 1B and C). The MLRs were much higher in the PD group than in the PR group $(P<0.05)$. These results suggested that lung cancer patients with higher MLRs obtained less benefit from rhES treatment and that peripheral monocytes may be an unfavorable factor for the effect of this drug.

Peripheral monocytes are the major source of macrophages in tumors. ${ }^{8}$ It has been shown that the circulating monocytes in blood may reflect the formation or presence of macrophages in tumors, ${ }^{18}$ and that there is a positive correlation between the MLR and macrophage count in the tumor microenvironment. ${ }^{19}$ So the clinical data described earlier suggest that macrophages play an important role in regulating the therapeutic effect of rhES. In order to clarify this issue, we assessed changes in macrophage numbers and polarization in the tumor microenvironment during rhES therapy. LLC xenograft mice were given NS or rhES for 14 days. Flow cytometry and immunofluorescence analyses were performed to determine the number of macrophages in LLC tumors. The results showed that the percentage of $\mathrm{F} 4 / 80^{+}$macrophages was increased significantly after 14 days of rhES treatment $(7.84 \% \pm 1.06 \%$ in the NS group vs $11.29 \% \pm 1.12 \%$ in the rhES group; $P<0.05$; Figure $2 \mathrm{~A}$ and B). Similar trends were observed in mice bearing A549 cell tumors (Figure S1).

The polarization of macrophages was determined by assessing the expression of $\mathrm{F} 4 / 80$ and specific markers of the subgroups (CD197 for the M1-like subgroup and CD206 for the M2-like subgroup). The results showed that the majority of macrophages in the rhES group exhibited M2-like phenotype $(P<0.05$; Figure $2 \mathrm{C})$.

To determine why rhES induced the accumulation of macrophages with M2-like phenotype, further experiments were performed to explore the potential mechanisms. The results have shown that rhES inhibited tumor angiogenesis

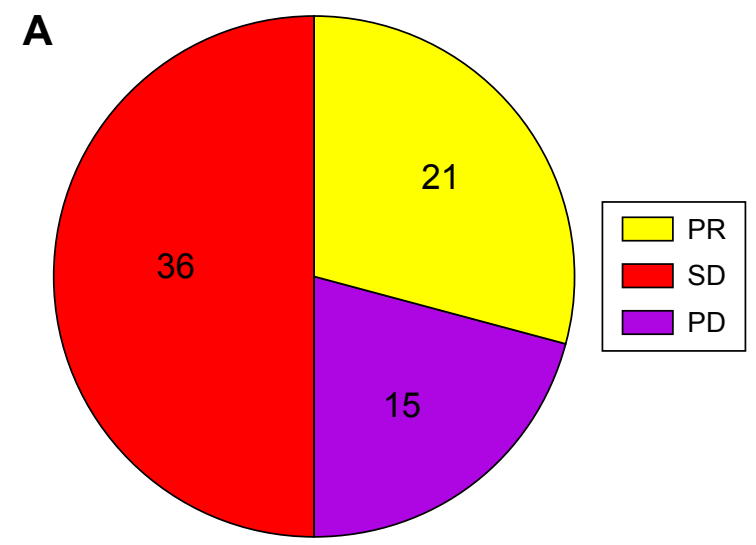

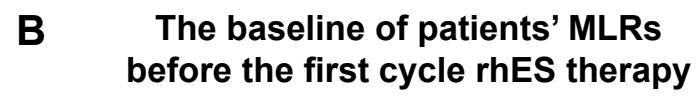

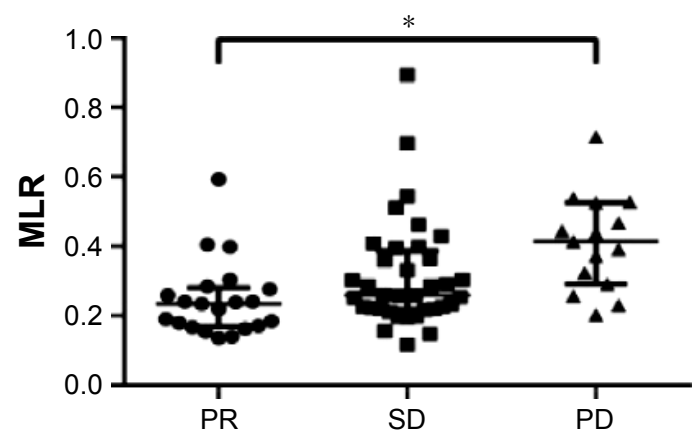

C

The baseline of patients' MLRs before the second cycle rhES therapy

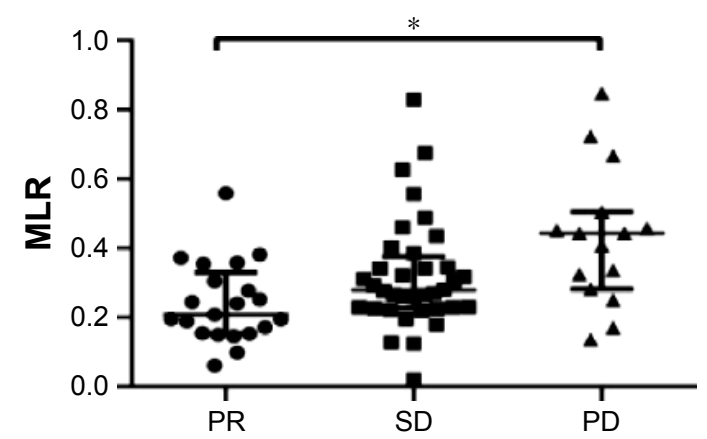

Figure I Lung cancer patients with higher monocyte-to-lymphocyte ratios (MLRs) obtained less benefit from rhES.

Notes: (A) The response of lung cancer patients to rhES therapy. (B) Baseline MLRs in patients before the first cycle of rhES therapy. (C) Baseline MLRs in patients before the second cycle of rhES therapy. Data are presented as the median \pm interquartile range. Kruskal-Wallis test was applied to assess statistical significance. $* P<0.05$. Abbreviations: PR, partial response; SD, stable disease; PD, progressive disease; MLRs, monocyte-to-lymphocyte ratios; rhES, recombinant human endostatin. 
A

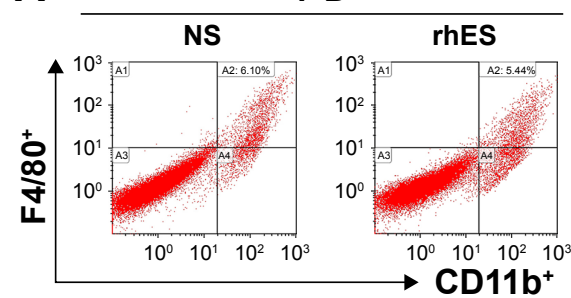

$14 \mathrm{D}$

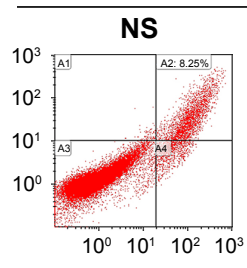

D

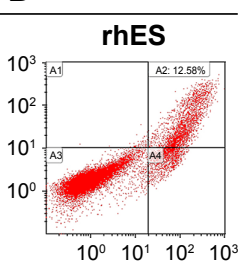

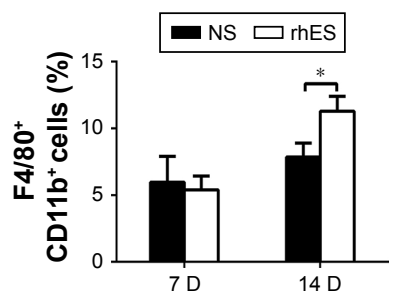

$\mathrm{F} 4 / 80^{+}$
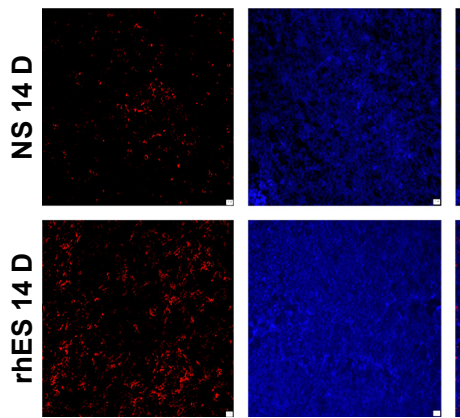

$7 \mathrm{D}$

C

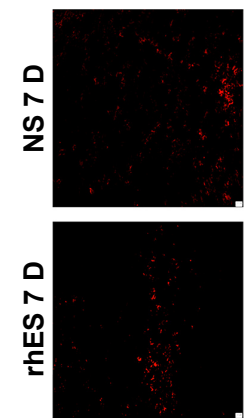

$7 \mathrm{D}$
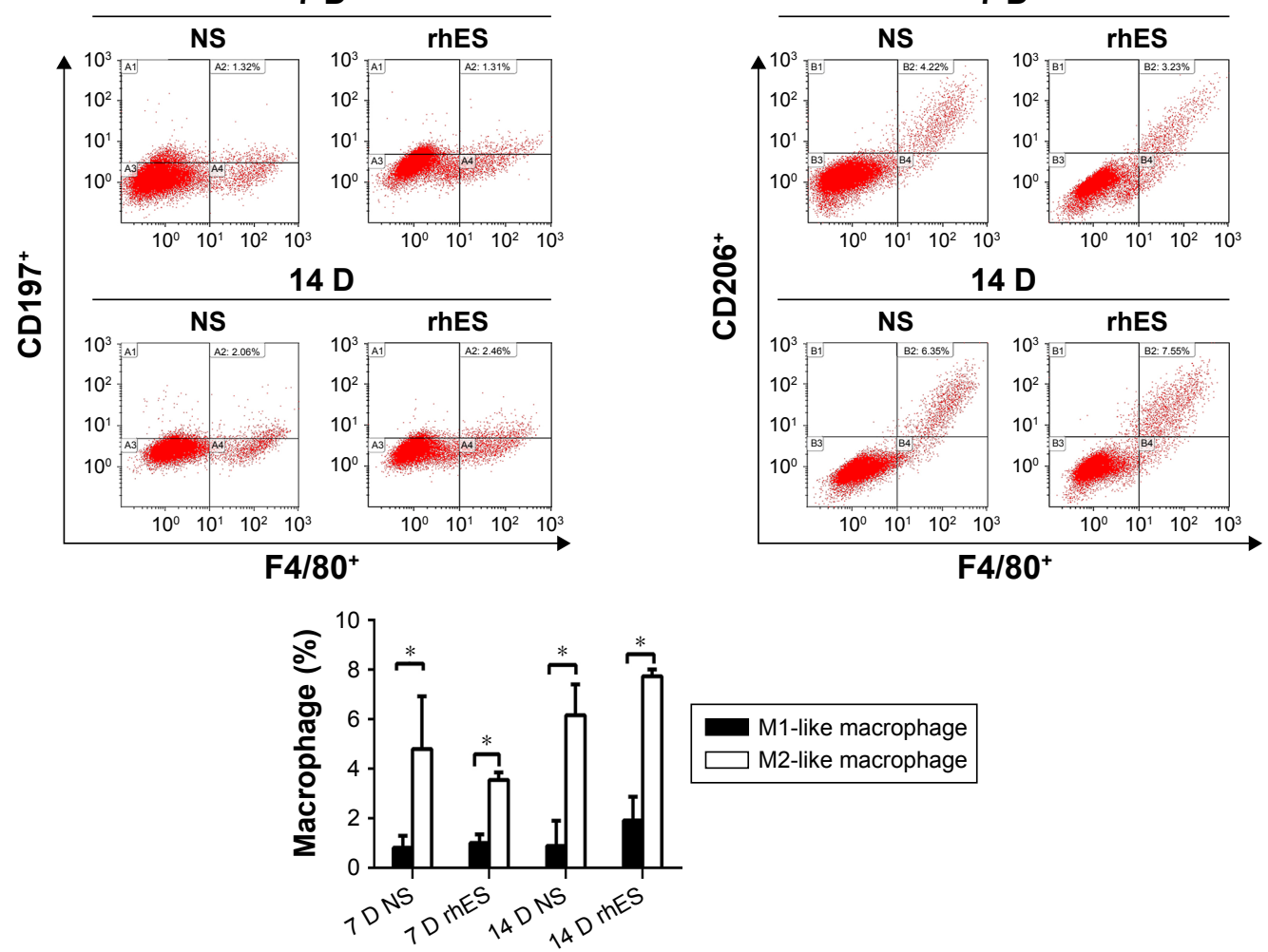

Figure 2 Macrophage accumulation in the tumor microenvironment increased after rhES therapy and the majority of them exhibited M2-like phenotype.

Notes: (A) The percentages of macrophages $\left(\mathrm{F} 4 / 80^{+} \mathrm{CDI} \mathrm{b}^{+}\right)$in Lewis lung carcinoma (LLC) tumors of mice treated with recombinant human endostatin (rhES) or normal saline (NS), 7 and 14 days after the initiation of treatment, were determined by flow cytometry. (B) Representative photomicrographs showing immunofluorescence

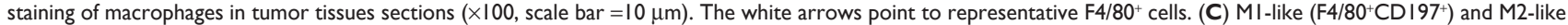
$\left(\mathrm{F} 4 / 80^{+} \mathrm{CD} 206^{+}\right)$macrophages were enumerated by flow cytometry. The majority exhibited an M2-like phenotype. Data are presented as mean \pm SD. $* P<0.05$.

Abbreviations: DAPI, 4',6-diamidino-2-phenylindole; D, days; SD, standard deviation.

and aggravated hypoxia and the inflammatory response in the tumor microenvironment. $\mathrm{CD} 31^{+}$cell numbers were reduced in the rhES group, indicating less angiogenesis in LLC tumors after rhES treatment (Figure 3A). VEGF levels in the tumor tissues were also decreased (Figure 3B). During treatment, increased hypoxic areas and number of necrotic/apoptotic cells were observed in the rhES group compared with the control group (Figure 3C and D). The levels of some inflammatory cytokines, such as IL-4 $(P<0.05)$, IL-6 $(P<0.05)$, and IL-10, were increased in the rhES group (Figure 3E). 
A

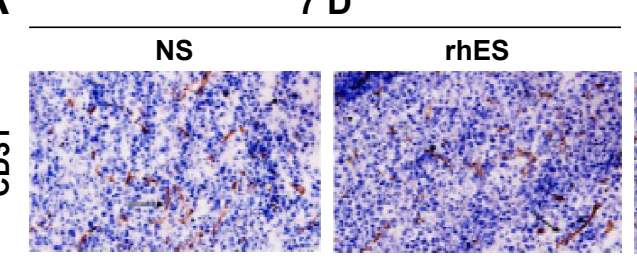

$7 \mathrm{D}$

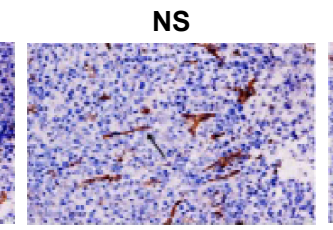

$14 \mathrm{D}$
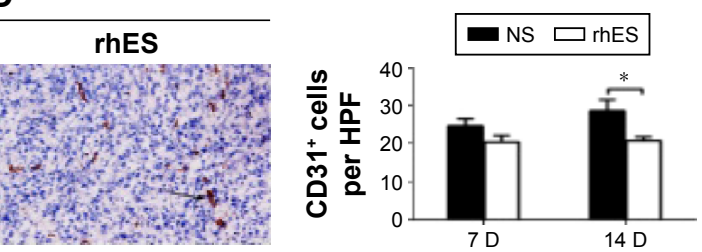

C
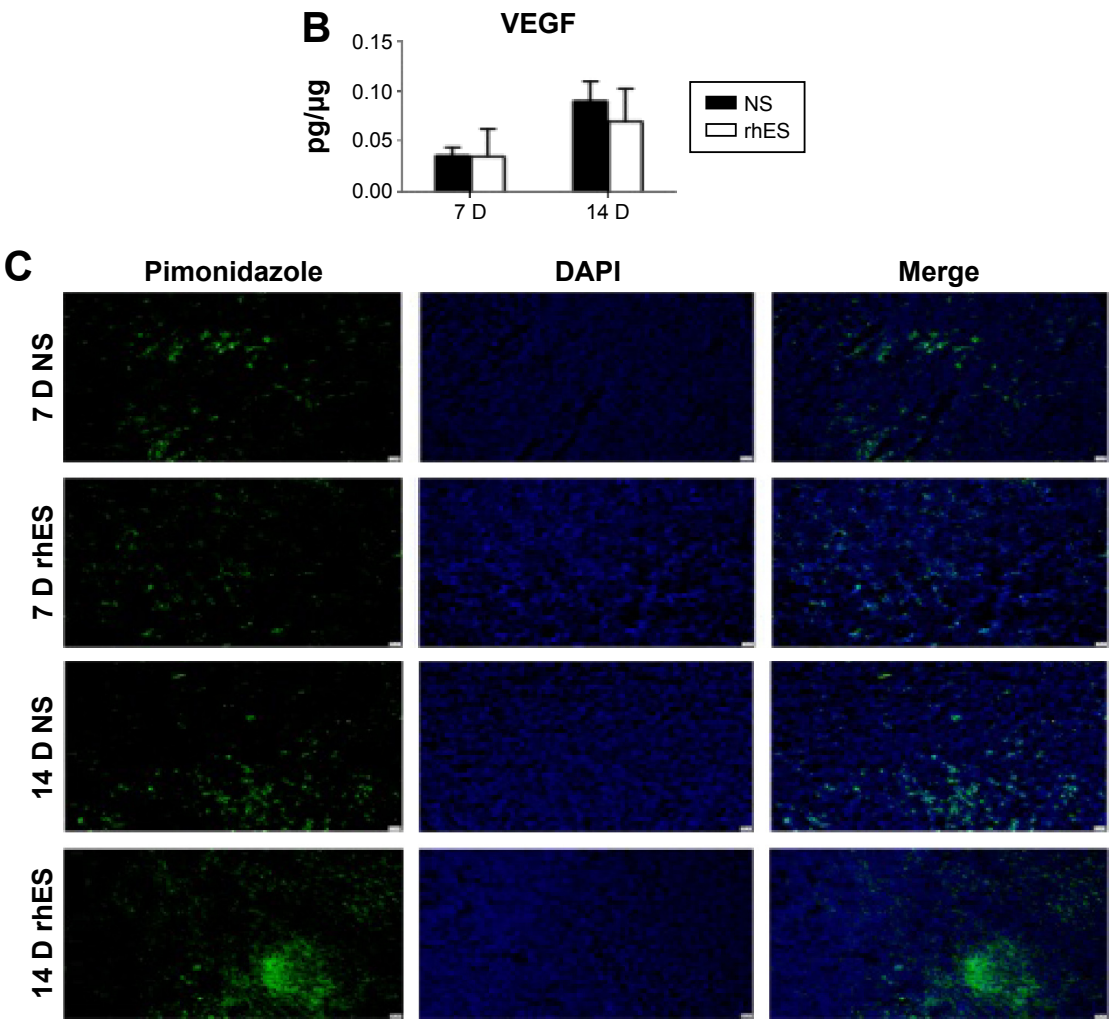

D

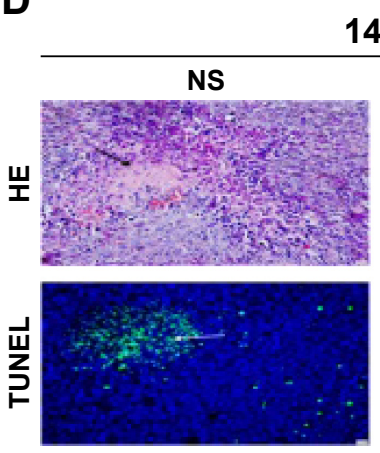

$14 \mathrm{D}$

E
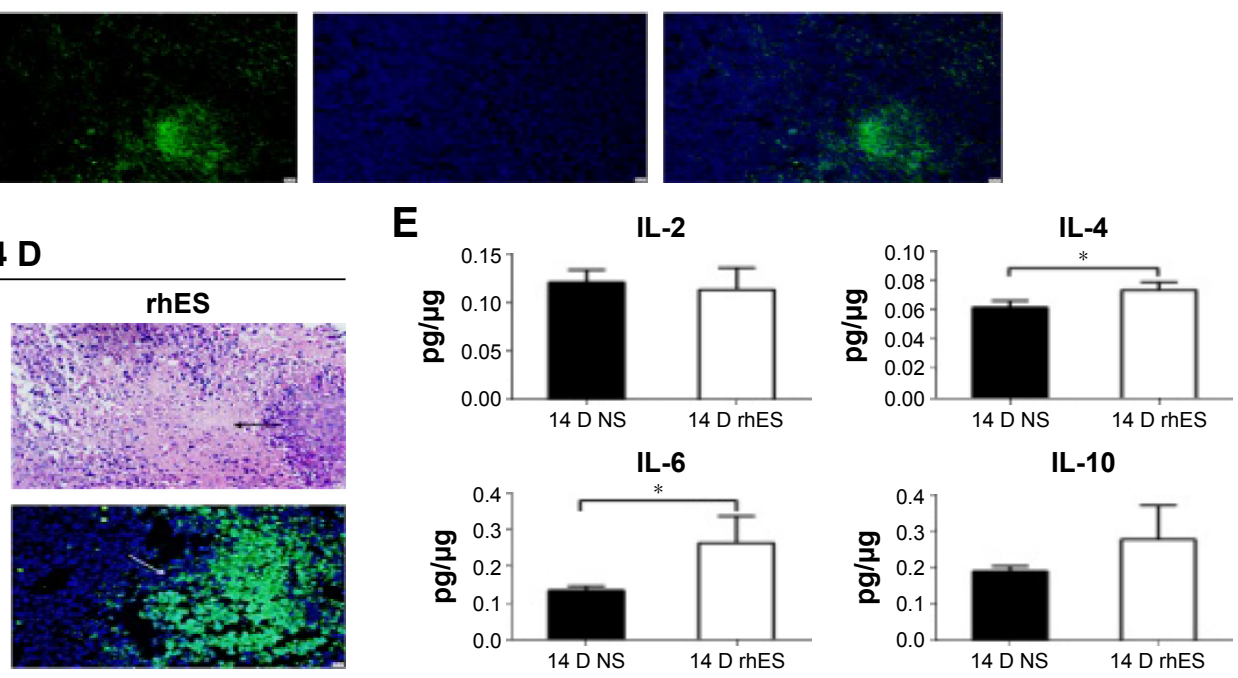

$\mathbf{F}$

$14 \mathrm{D}$

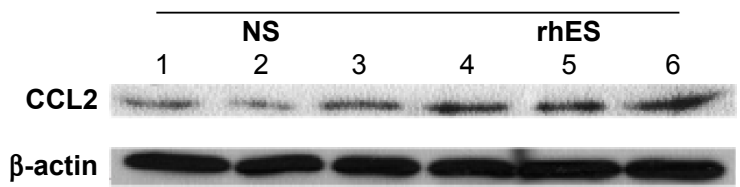

Figure 3 rhES aggravated hypoxia and the inflammatory response in the tumor microenvironment.

Notes: (A) Photographs showing the CD3I immunohistochemistry in Lewis lung carcinoma (LLC) tumors at 7 and I4 days after the initiation of treatment $(\times 200$, scale bar $=10 \mu \mathrm{m}$ ). (B) Levels of vascular endothelial growth factor (VEGF) in LLC tumors were detected by an enzyme-linked immunosorbent assay (ELISA). (C) Hypoxic areas in LLC tumors were visualized by immunolabeling for the hypoxia-specific marker pimonidazole. (D) After I4 days of rhES treatment, necrotic or apoptotic cells in LLC tumor tissues were visualized by hematoxylin and eosin or terminal deoxynucleotidyl transferase dUTP nick end labeling $(T U N E L)$ staining, respectively $(\times 200$, scale bar $=10 \mu \mathrm{m})$. (E) Levels of IL-2, IL-4, IL-6, and IL- I 0 in LLC tumors were detected by an ELISA. (F) Levels of CCL2 in LLC tumors were analyzed by Western blot. Samples I, 2, and 3 were taken from tumors in the normal saline (NS) group. Samples 4,5 , and 6 were taken from tumors in the recombinant human endostatin (rhES) group. Data are presented as mean \pm SD. $* P<0.05$. Abbreviations: IL, interleukin; SD, standard deviation; DAPI, 4',6-diamidino-2-phenylindole; D, days. 
Levels of CCL2, a chemotactic factor, were higher in the tumor samples from the rhES group (Figure 3F).

CCL 2 can recruit monocytes and macrophages. ${ }^{20}$ In vitro experiments demonstrated that hypoxia enhanced CCL2 expression in endothelial cells and fibroblasts. $\mathrm{CoCl}_{2}$, a chemical hypoxia-inducing agent, is frequently used and widely accepted to mimic hypoxia in vitro. ${ }^{21}$ Levels of CCL2 were increased in HUVECs and HSF cells treated with $\mathrm{CoCl}_{2}$ for 24,48 , and 72 hours (Figure $4 \mathrm{~A}$ ). The mechanism of this effect may be associated with the activation of ERK1/2 via hypoxia (Figure S2). The transwell assay demonstrated that CCL2 attracted macrophages. When CCL2 was added to the lower chamber, more number of myeloid monocytes, as well as RAW264.7 and ANA-1 cells, migrated to the lower chamber (Figure 4B).

Inflammatory cytokines can affect macrophage polarization. ${ }^{22}$ Because IL-4, IL-6, and IL-10 levels increased in tumor tissues after rhES treatment, BMDMs were cultured with these ILs for 96 hours. Flow cytometry results showed that the percentage of $\mathrm{CD} 86^{+}$cells (M1-like) was only slightly elevated. However, the percentage of CD206 ${ }^{+}$cells (M2-like) was significantly increased $(24.9 \% \pm 3.6 \%$ in the control group vs $79.7 \% \pm 6.3 \%$ in the IL-4 group, $78.3 \% \pm 0.27 \%$ in the IL-6 group, and $38.1 \% \pm 5.4 \%$ in the IL-10 group; $P<0.05$, Figure 4C). The expression of M2-like marker mRNA was also examined. The mRNA levels of ARG1 and YM1 presented an increasing trend in macrophages induced by these inflammatory cytokines, compared with those in the matched control group (Figure 4D). Similarly, when the RAW264.7 and ANA-1 macrophage cell lines were stimulated with IL-4, IL-6, or IL-10, the CD206 ${ }^{+}$subgroups also increased significantly $(P<0.05$; Figure $4 \mathrm{E})$.

Evidence suggests that highly metastatic subgroups and stem-like cancer cells are enriched during anti-angiogenic therapy. This is an important reason that could possibly explain how tumor cells could escape from this therapeutic treatment. However, the mechanism of this effect is not fully understood..$^{23,24}$ In the current study, we found that macrophages induced by hypoxia or inflammatory cytokines may contribute to this process. After incubation in $1 \% \mathrm{O}_{2}$ for 24 hours, macrophages (BMDMs and RAW264.7 cells) expressed more amounts of MMP-9 and VEGF than control cells (Figure 5A and B).

After culturing with inflammatory cytokines, macrophages showed the ability to enhance LLC progression. We cultured BMDMs in the presence of IL-4, IL6, or IL-10 for 96 hours. The CM obtained from these cultures increased the percentage of ALDH (a marker of lung cancer stem cells) positive
LLC cells, enhanced LLC cell migration, and induced the epithelial-mesenchymal transition (Figure $5 \mathrm{C}-\mathrm{F}$ ). The results of in vivo experiments were consistent with our in vitro results. When LLC cells were cultured for 48 hours in CM from macrophages exposed to IL-4 or hypoxia and injected into mice via the tail vein, more metastatic nodes formed in the +IL4-CM and Hypoxia-CM groups than in the control group after 3 weeks $(P<0.05$; Figure $5 \mathrm{G})$.

\section{Discussion}

In this study, we found that although rhES inhibited lung cancer angiogenesis, it aggravated hypoxia and the inflammatory response in the tumor microenvironment, including increases in the levels of IL-4, IL-6, IL-10, and CCL2. These changes were favorable for macrophage accumulation and skewed their polarization toward the M2-like phenotype which could enhance tumor progression, leading to tumor escape from anti-angiogenic therapy. Thus, our results indicate that the accumulation of macrophages may adversely affect the efficacy of rhES treatment. In addition, as there is a positive correlation between the macrophage count in tumors and monocyte percentage in peripheral blood, this conclusion may also explain why peripheral monocyte levels are associated with the outcome of rhES in the clinical setting.

Recent evidence has shown that, despite the widespread use of anti-angiogenic drugs for cancer treatment, their effects remain limited because of intrinsic tumor refractoriness and the emergence of treatment-induced resistance. ${ }^{4}$ Other studies suggest that some bone marrow-derived cells, such as $\mathrm{CD} 1 \mathrm{~b}^{+} \mathrm{Grl}^{+}$myeloid cells, in the tumor microenvironment are associated with tumor escape from antiangiogenesis therapy and contribute to tumor refractoriness to anti-VEGF agents. ${ }^{25,26}$

Macrophages are a component of bone marrow-derived cells that play important roles in inflammation, immunity, and tumor progression. ${ }^{9}$ Some studies suggest that macrophages accumulate in glioma after bevacizumab therapy, and a high level of macrophages predicts a poor outcome. ${ }^{27}$ In the present study, we found that macrophage accumulation in LLC tumors increased after rhES treatment. We speculated that this was related to increases in CCL2, a major chemoattractant factor for macrophages and monocytes. ${ }^{20}$ Hypoxia has been reported to be associated with the expression of CCL2 in tumors. ${ }^{28}$ Our results showed that rhES aggravated hypoxia in the tumor microenvironment, and that hypoxia promoted the expression of CCL2 in endothelial and fibroblast cells. The mechanism of this effect was associated with the activation of ERK1/2 via hypoxia (Figure S2). 


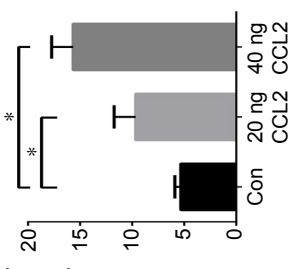

$\left.{ }_{1} \mathrm{OLX}\right)$ ләшечо ләмо। ә૫¥ U! دәqunu ॥əכ

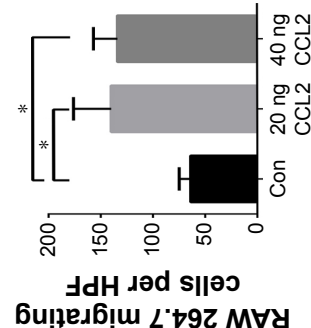

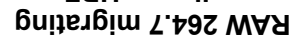

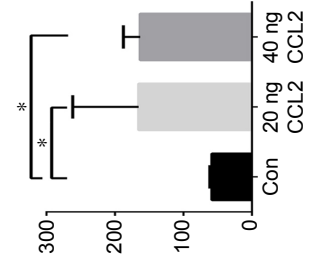

JdH גəd s\|əo

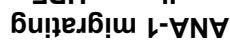

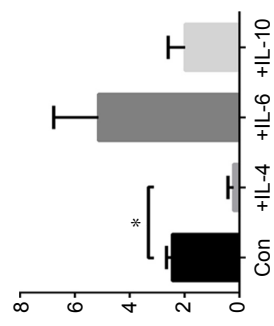

(\%) s||ə0 +980ว

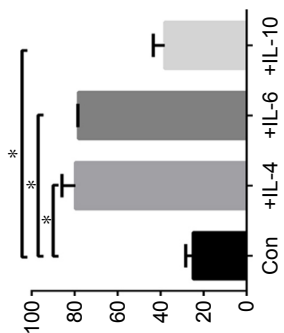

(\%) s||əo +90Z0ว
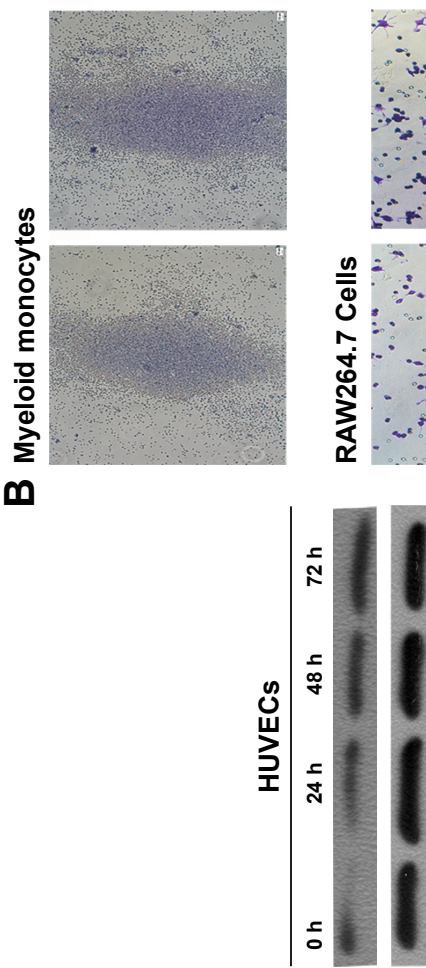

ฮั่ำ ำ

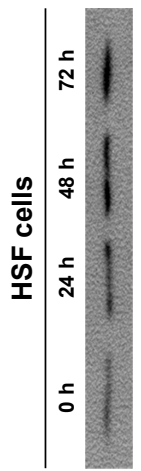

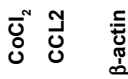
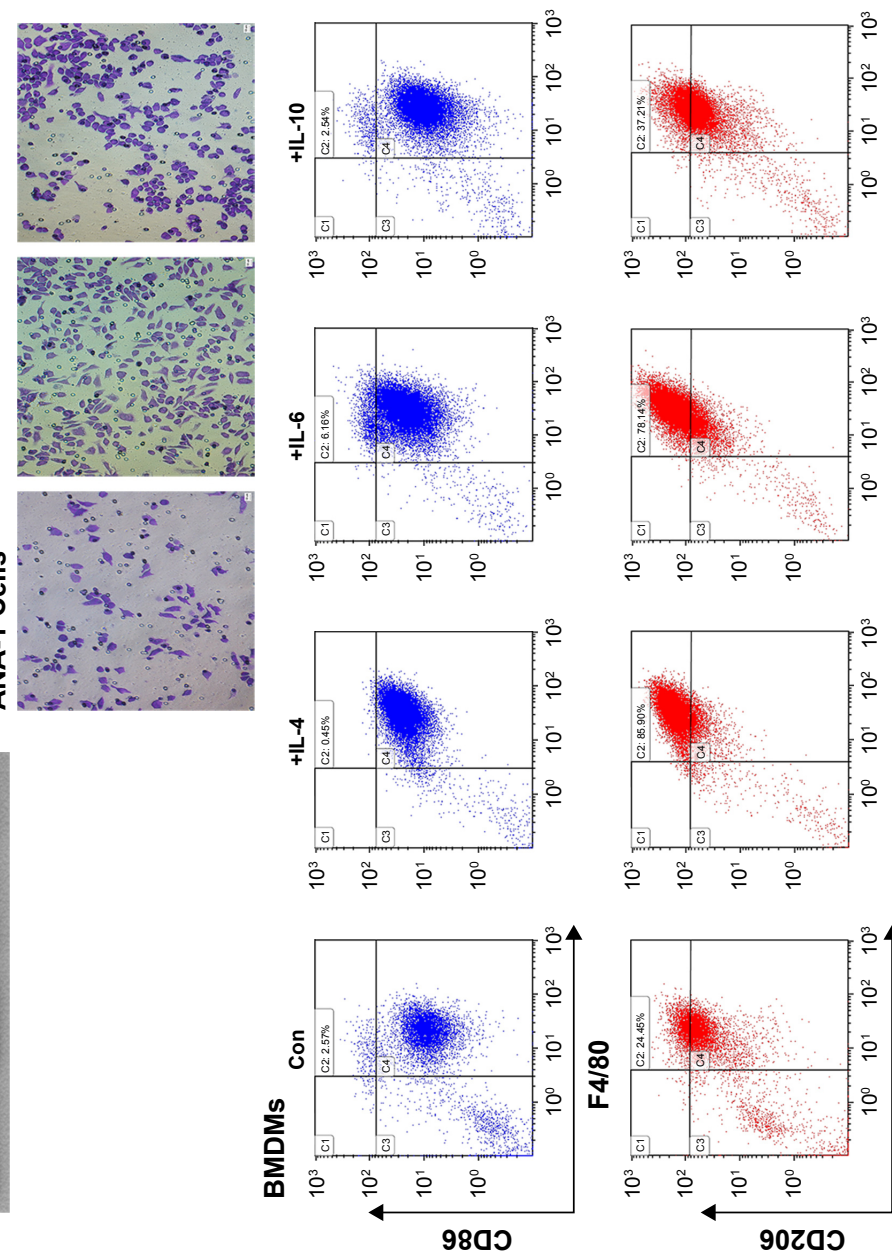

U

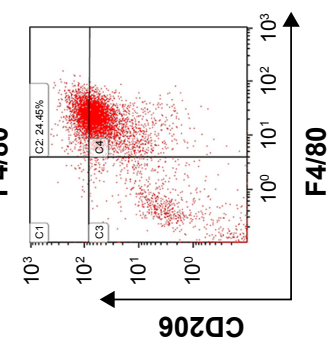



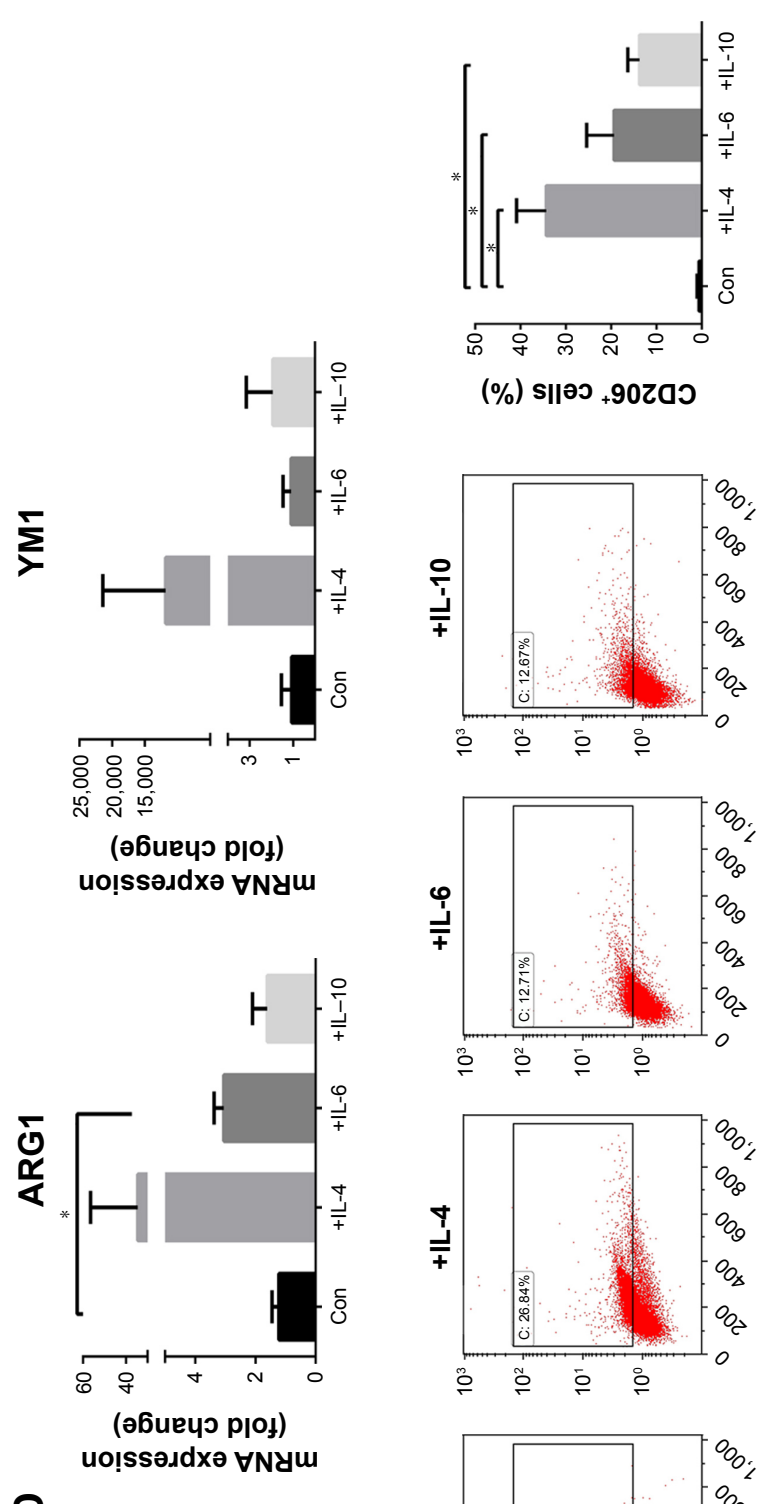

口
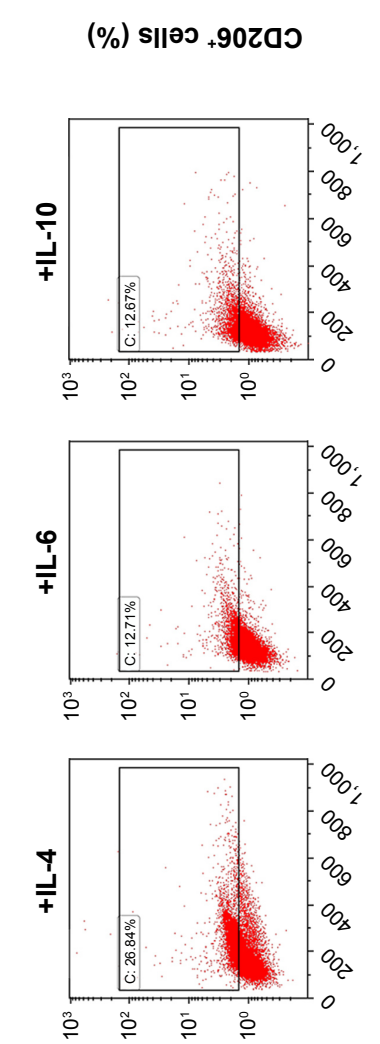

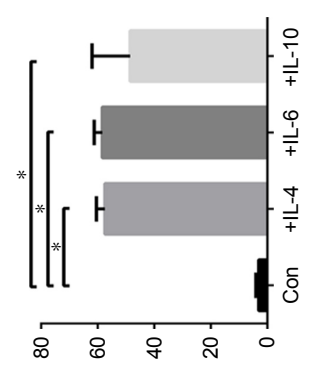

(\%) s||ə0 +90Z0ว
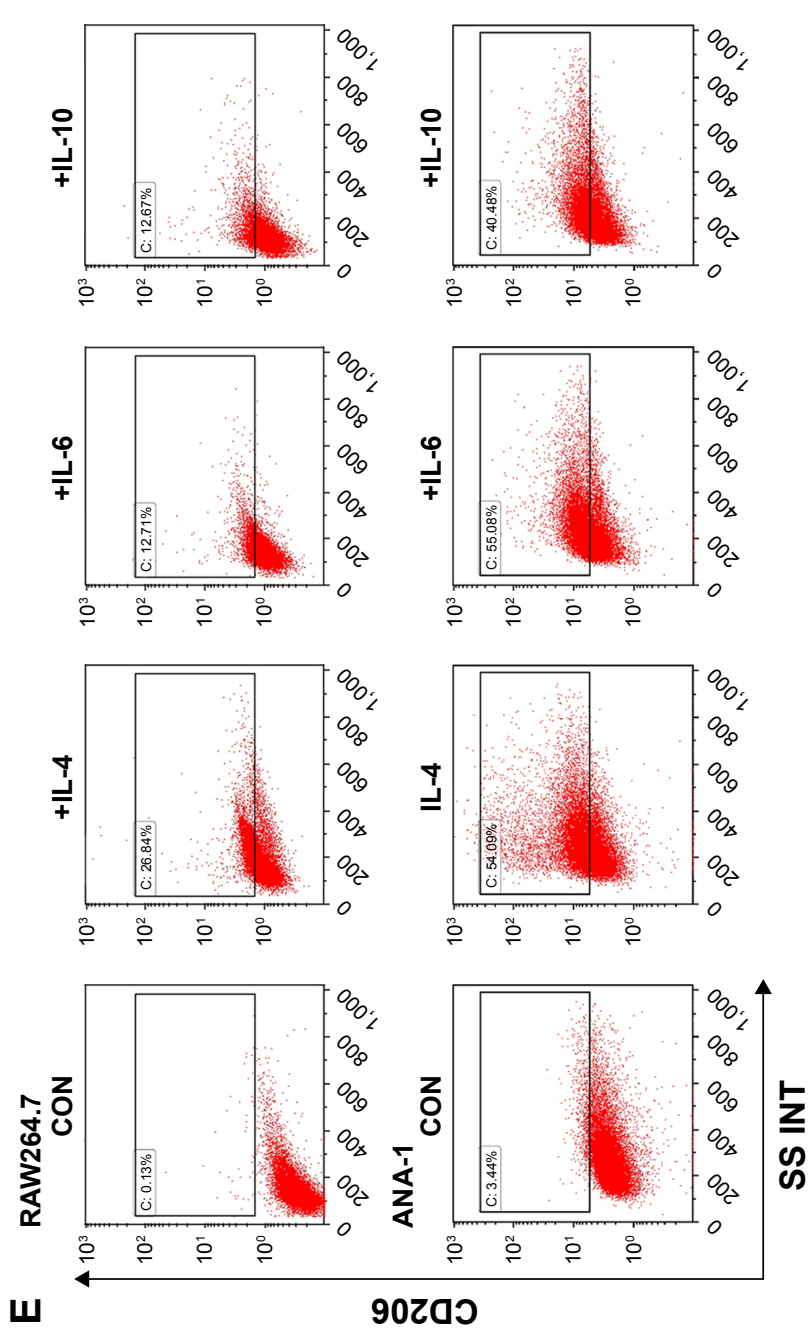

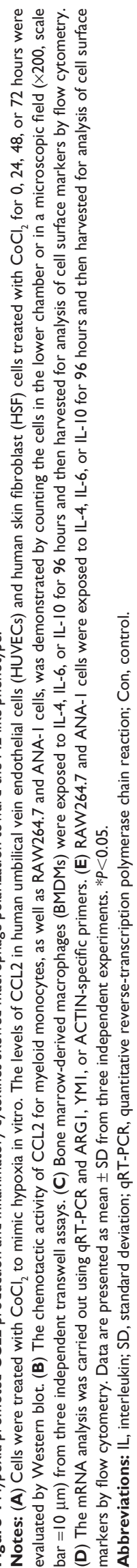


A
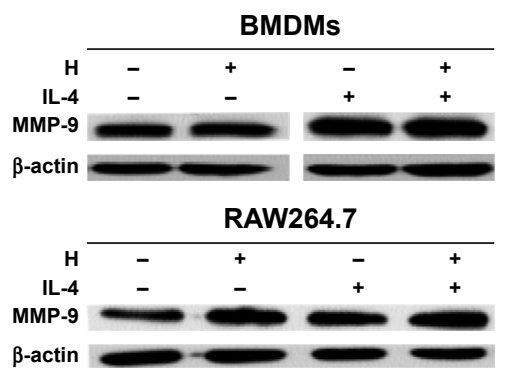

B

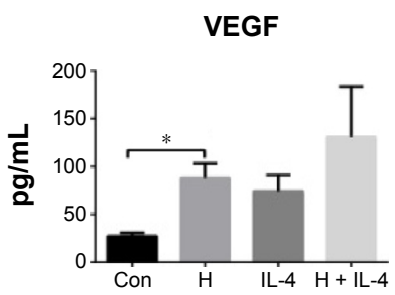

C
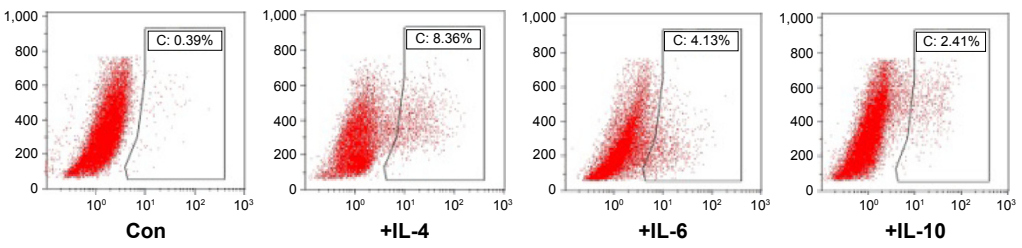

BMDMs conditioned media

D

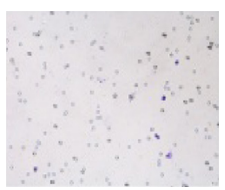

Con

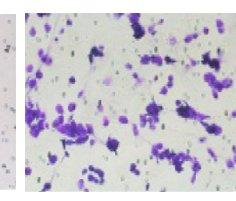

+ IL-4

BMDMs conditioned media

E
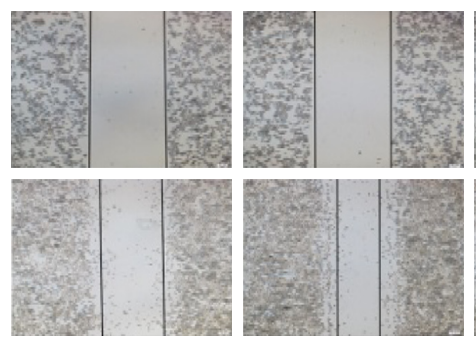

$+\mathrm{IL}-4$

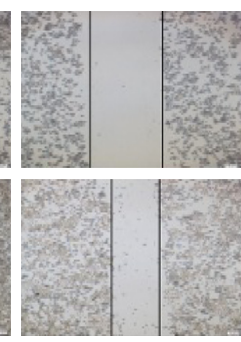

+IL-6

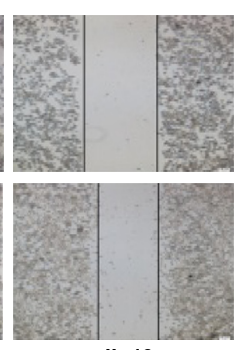

+ IL-10

BMDMs conditioned media

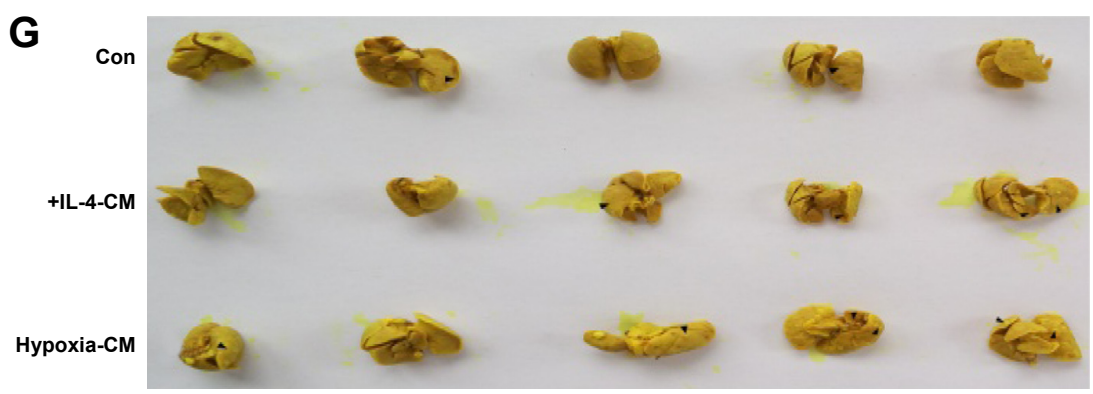

RAW264.7
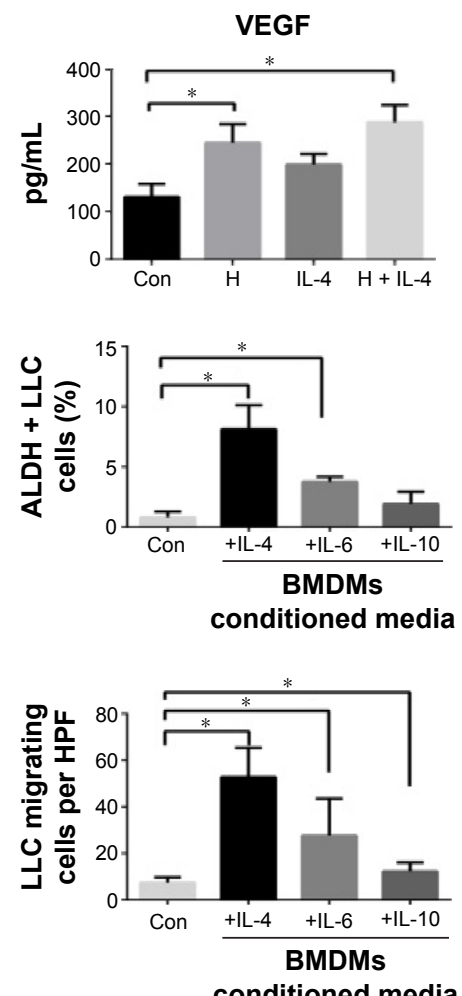

$\mathbf{F}$

+IL-4 BMDMs conditioned media

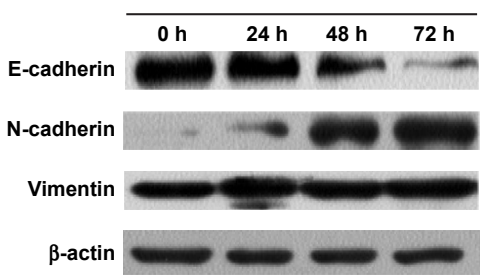

Figure 5 Macrophages treated with hypoxia or inflammatory cytokines promoted the progression of LLC.

Notes: (A) Levels of MMP-9 in BMDMs and RAW264.7 cells under hypoxia $\left(1 \% \mathrm{O}_{2}\right)$ or in the presence of IL-4 were detected by Western blot. (B) Levels of VEGF in the supernatant of BMDMs and RAW264.7 cells under hypoxia or in the presence of IL-4 were detected by ELISA. (C) Conditioned media (CM) were collected from BMDMs subjected to hypoxia or in the presence of interleukins. LLC cells were incubated with the indicated CM for 48 hours and the proportion of aldehyde dehydrogenase positive $\left(\mathrm{ALDH}^{+}\right)$LLC cells were analyzed by flow cytometry. (D) LLC cells were incubated with the indicated CM using a 24-well transwell chamber for 24 hours and the number of migrating cells was determined. (E) A wound healing assay was used to examine the effect of activated macrophages on the migration of LLC cells. (F) LLC cells were incubated with the indicated CM for 24,48 , or 72 hours and the levels of E-cadherin, N-cadherin, and vimentin were evaluated by Western blot. (G) LLC cells were incubated with the indicated CM for 48 hours and then injected into the tail vein of C57BL/6 mice. Mice were assigned to three groups of five each: control, +IL4-CM, and Hypoxia-CM. Photographs of lung metastases were used for the quantitative analysis of lung metastatic nodules. Data are presented as mean \pm SD from three independent experiments. $* P<0.05$. Abbreviations: BMDMs, bone marrow-derived macrophages; LLC, Lewis lung carcinoma; IL, interleukin; VEGF, vascular endothelial growth factor; ELISA, enzyme-linked immunosorbent assay; SD, standard deviation; HPF, high-power field; Con, control. 
Macrophages can be divided into different subgroups. Depending on their polarization, there are two well-established phenotypes: classically activated macrophages (M1) and alternatively activated macrophages (M2). It is generally considered that these two types of macrophages are antagonistic. M1-like subgroup macrophages inhibit tumor growth, whereas the M2-like subgroup macrophages tend to promote tumor progression and can lead to tumor escape from anti-angiogenic therapy. ${ }^{4,29}$ In addition, some Tie $2^{+}$ macrophages/monocytes, which enhance angiogenesis, are observed in tumors. ${ }^{30}$ In the current study, we found that the number of $\mathrm{Tie} 2^{+}$macrophages were not statistically significantly different between the control and rhES groups (Figure S3), and most macrophages in the tumors treated with rhES were of the M2-like phenotype. In order to clarify the mechanism that increased M2-like macrophage numbers in tumors treated with rhES, we focused on changes in inflammatory cytokines in the tumor microenvironment, because our previous study had demonstrated that rhES induced an inflammatory response in tumors. ${ }^{14}$ The results showed that IL-4, IL-6, and IL-10 levels were increased and that they can skew macrophage polarization. ${ }^{31}$ In vitro, we observed that when induced by IL-4, IL-6, or IL-10, macrophages showed a high expression of CD206 and enhanced the progression of LLC. Among these interleukins, IL-4 and IL-6 presented a stronger effect on regulating macrophage polarization and function than IL-10.

Since shifts in the macrophage phenotype are highly associated with the environment, ${ }^{22}$ we speculate that changes in the tumor microenvironment mediated by rhES are a main cause for the accumulation of macrophages and skewing their polarization toward the M2-like phenotype. However, the effect of anti-angiogenic therapy on the tumor microenvironment remains incompletely understood, and some results have led to inconsistent conclusions. For example, some evidence shows that anti-angiogenic therapy increases tumor hypoxia, ${ }^{23}$ while other results inferred that anti-angiogenic agents could lead to tumor vascular normalization and attenuated tumor hypoxia for a short time. ${ }^{32}$ Huang et al suggested that the effect of anti-angiogenic agents on reprogramming the tumor microenvironment depended on the drug dosage. ${ }^{33}$ In addition, different tumors may show different responses to anti-angiogenic therapy. We showed previously that rhES treatment aggravated the inflammatory response in B16 tumor-bearing mice, but not in CT26 tumor-bearing mice. ${ }^{11}$ Furthermore, treatment modalities may lead to different changes in the tumor microenvironment. For example, amplification of the endostatin gene in macrophages skewed their polarization to the M1 phenotype and resulted in delayed breast cancer growth in vivo. ${ }^{34}$ This result suggested that, compared with rhES, gene therapy may perform functions in addition to inhibiting angiogenesis. Because the issue is complex, further preclinical experiments and clinical trial evidence are required to help us understand the interaction between anti-angiogenic therapy, the tumor microenvironment, and macrophages.

\section{Conclusion}

In summary, our data indicate that rhES could aggravate hypoxia and the inflammatory response in the tumor microenvironment. These changes could induce macrophage accumulation, and skewed their polarization toward the M2-like phenotype, which could help LLC to escape from the anti-angiogenic therapy. Therefore, the accumulation of macrophages in the tumor microenvironment may attenuate the effect of rhES treatment on lung cancer. In the future, combination therapy that also targets macrophages or their relevant factors may improve the efficacy of anti-angiogenic therapy.

\section{Acknowledgments}

This study was supported by grants from the National Natural Science Foundation of China (Grant nos 81372506 and 81401897).

\section{Disclosure}

The authors report no conflicts of interest in this work.

\section{References}

1. Jemal A, Bray F, Center MM, Ferlay J, Ward E, Forman D. Global cancer statistics. CA Cancer J Clin. 2011;61(2):69-90.

2. Rengan R, Maity AM, Stevenson JP, Hahn SM. New strategies in nonsmall cell lung cancer: improving outcomes in chemoradiotherapy for locally advanced disease. Clin Cancer Res. 2011;17(13):4192-4199.

3. Neri D, Bicknell R. Tumour vascular targeting. Nat Rev Cancer. 2005; 5(6):436-446.

4. Shojaei F. Anti-angiogenesis therapy in cancer: current challenges and future perspectives. Cancer Lett. 2012;320(2):130-137.

5. Bao Y, Peng F, Zhou QC, et al. Phase II trial of recombinant human endostatin in combination with concurrent chemoradiotherapy in patients with stage III non-small-cell lung cancer. Radiother Oncol. 2015;114(2): $161-166$

6. Ribatti D, Crivellato E, Vacca A. Inflammation and antiangiogenesis in cancer. Curr Med Chem. 2012;19(7):955-960

7. Botta C, Barbieri V, Ciliberto D, et al. Systemic inflammatory status at baseline predicts bevacizumab benefit in advanced non-small cell lung cancer patients. Cancer Biol Ther. 2013;14(6):469-475.

8. Go SI, Kim RB, Song HN, et al. Prognostic significance of the lymphocyte-to-monocyte ratio in patients with small cell lung cancer. Med Oncol. 2014;31(12):323.

9. Ruffell B, Coussens LM. Macrophages and therapeutic resistance in cancer. Cancer Cell. 2015;27(4):462-472. 
10. Condamine T, Ramachandran I, Youn JI, Gabrilovich DI. Regulation of tumor metastasis by myeloid-derived suppressor cells. Annu Rev Med. 2015;66:97-110.

11. Zhang $H$, Wang Z, Peng Q, et al. Tumor refractoriness to endostatin antiangiogenesis is associated with the recruitment of CD11b+Gr1+ myeloid cells and inflammatory cytokines. Tumori. 2013;99(6):723-733.

12. Anzinger JJ, Chang J, Xu Q, et al. Murine bone marrow-derived macrophages differentiated with GM-CSF become foam cells by PI3K $\gamma$-dependent fluid-phase pinocytosis of native LDL. J Lipid Res. 2012;53(1):34-42.

13. Zhang J, Cao J, Ma S, et al. Tumor hypoxia enhances non-small cell lung cancer metastasis by selectively promoting macrophage M2 polarization through the activation of ERK signaling. Oncotarget. 2014;5(20):9664-9677.

14. Wang Z, Li Z, Wang Y, et al. Versican silencing improves the antitumor efficacy of endostatin by alleviating its induced inflammatory and immunosuppressive changes in the tumor microenvironment. Oncol Rep. 2015;33(6):2981-2991

15. Gaur P, Sceusi EL, Samuel S, et al. Identification of cancer stem cells in human gastrointestinal carcinoid and neuroendocrine tumors. Gastroenterology. 2011;141(5):1728-1737.

16. Ljungkvist AS, Bussink J, Kaanders JH, et al. Hypoxic cell turnover in different solid tumor lines. Int J Radiat Oncol Biol Phys. 2005;62(4): 1157-1168.

17. Rashid OM, Nagahashi M, Ramachandran S, et al. Is tail vein injection a relevant breast cancer lung metastasis model? J Thorac Dis. 2013; 5(4):385-392.

18. Szkandera J, Gerger A, Liegl-Atzwanger B, et al. The lymphocyte/ monocyte ratio predicts poor clinical outcome and improves the predictive accuracy in patients with soft tissue sarcomas. Int J Cancer. 2014; 135(2):362-370.

19. Koh YW, Kang HJ, Park C, et al. The ratio of the absolute lymphocyte count to the absolute monocyte count is associated with prognosis in Hodgkin's lymphoma: correlation with tumor-associated macrophages. Oncologist. 2012;17(6):871-880.

20. Zhang J, Lu Y, Pienta KJ. Multiple roles of chemokine (C-C motif) ligand 2 in promoting prostate cancer growth. J Natl Cancer Inst. 2010; 102(8):522-528

21. Zhang $\mathrm{H}, \mathrm{Wu} \mathrm{H}$, Zheng $\mathrm{J}$, et al. Transforming growth factor $\beta 1$ signal is crucial for dedifferentiation of cancer cells to cancer stem cells in osteosarcoma. Stem Cells. 2013;31(3):433-446.
22. Ivashkiv LB. Epigenetic regulation of macrophage polarization and function. Trends Immunol. 2013;34(5):216-223.

23. Pàez-Ribes M, Allen E, Hudock J, et al. Antiangiogenic therapy elicits malignant progression of tumors to increased local invasion and distant metastasis. Cancer Cell. 2009;15(3):220-231.

24. Wang Y, Jiang M, Li Z, et al. Hypoxia and TGF-beta1 lead to endostatin resistance by cooperatively increasing cancer stem cells in A549 transplantation tumors. Cell Biosci. 2015;5:72.

25. Shojaei F, Wu X, Malik AK, et al. Tumor refractoriness to anti-VEGF treatment is mediated by CD11b+Gr1+ myeloid cells. Nat Biotechnol. 2007;25(8):911-920.

26. de Groot JF, Piao Y, Tran H, et al. Myeloid biomarkers associated with glioblastoma response to anti-VEGF therapy with aflibercept. Clin Cancer Res. 2011;17(14):4872-4881.

27. Lu-Emerson C, Snuderl M, Kirkpatrick ND, et al. Increase in tumorassociated macrophages after antiangiogenic therapy is associated with poor survival among patients with recurrent glioblastoma. NeuroOncol. 2013;15(8):1079-1087.

28. Tao LL, Shi SJ, Chen LB, Huang GC. Expression of monocyte chemotactic protein-1/CCL2 in gastric cancer and its relationship with tumor hypoxia. World J Gastroenterol. 2014;20(15):4421-4427.

29. Sica A, Mantovani A. Macrophage plasticity and polarization: in vivo veritas. J Clin Invest. 2012;122(3):787-795.

30. De Palma M, Venneri MA, Galli R, et al. Tie2 identifies a hematopoietic lineage of proangiogenic monocytes required for tumor vessel formation and a mesenchymal population of pericyte progenitors. Cancer Cell. 2005;8(3):211-226.

31. Van Dyken SJ, Locksley RM. Interleukin-4- and interleukin-13mediated alternatively activated macrophages: roles in homeostasis and disease. Annu Rev Immunol. 2013;31:317-343.

32. Carmeliet P, Jain RK. Principles and mechanisms of vessel normalization for cancer and other angiogenic diseases. Nat Rev Drug Discov. 2011; 10(6):417-427.

33. Huang Y, Yuan J, Righi E, et al. Vascular normalizing doses of antiangiogenic treatment reprogram the immunosuppressive tumor microenvironment and enhance immunotherapy. Proc Natl Acad Sci U S A. 2012;109(43):17561-17566.

34. Guo H, Liu Y, Gu J, et al. Endostatin inhibits the growth and migration of 4T1 mouse breast cancer cells by skewing macrophage polarity toward the M1 phenotype. Cancer Immunol Immunother. Epub 2016 Mar 31. 


\section{Supplementary materials}

$12 \mathrm{D}$

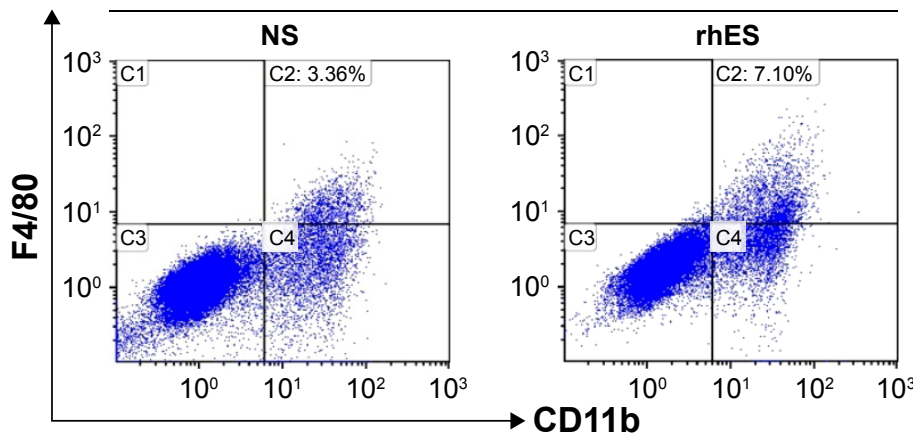

$12 \mathrm{D}$

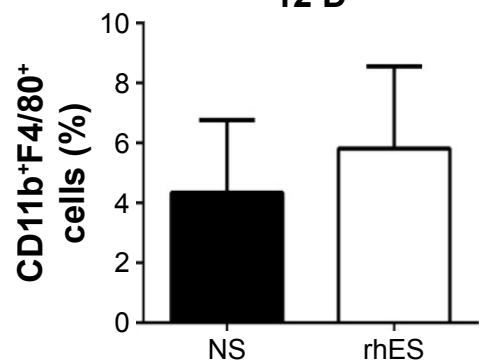

Figure SI Macrophages in tumor tissues of A549 cell tumor-bearing mice treated with recombinant human endostatin (rhES) for 12 days. A549 cell tumor-bearing mice were treated with normal saline (NS) or rhES for 12 days. The number of macrophages were counted by flow cytometry. Data are presented as mean \pm SD ( $=4-5$ animals per group).

Abbreviations: SD, standard deviation; D, days.

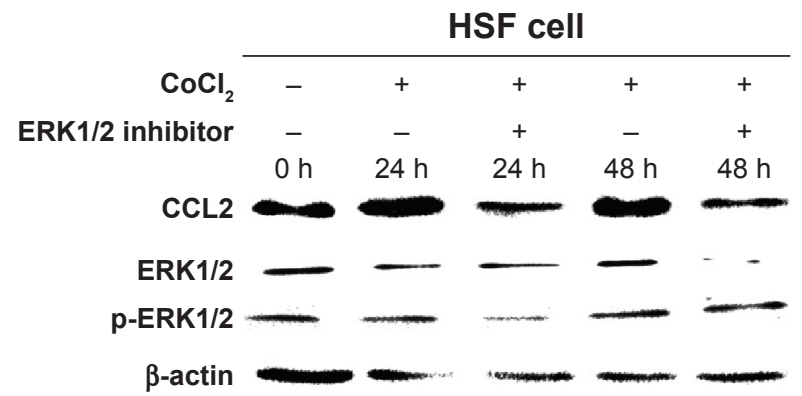

Figure S2 CCL2 expression levels in HSF cells under hypoxia with or without an ERKI/2 inhibitor. CoCl 2 was used to mimic hypoxia in vitro. SCH772984 (Selleck Chemicals, Houston, TX, USA) is an ERKI/2 inhibitor. Human skin fibroblast (HSF) cells were treated with CoCl ${ }_{2}$ with or without SCH772984 (5 $\mu$ M) for 24 or 48 hours. The levels of CCL2, ERKI/2, and p-ERKI/2 (all from Zen-Bioscience) in HSF cells were evaluated by Western blot.

\section{$14 \mathrm{D}$}
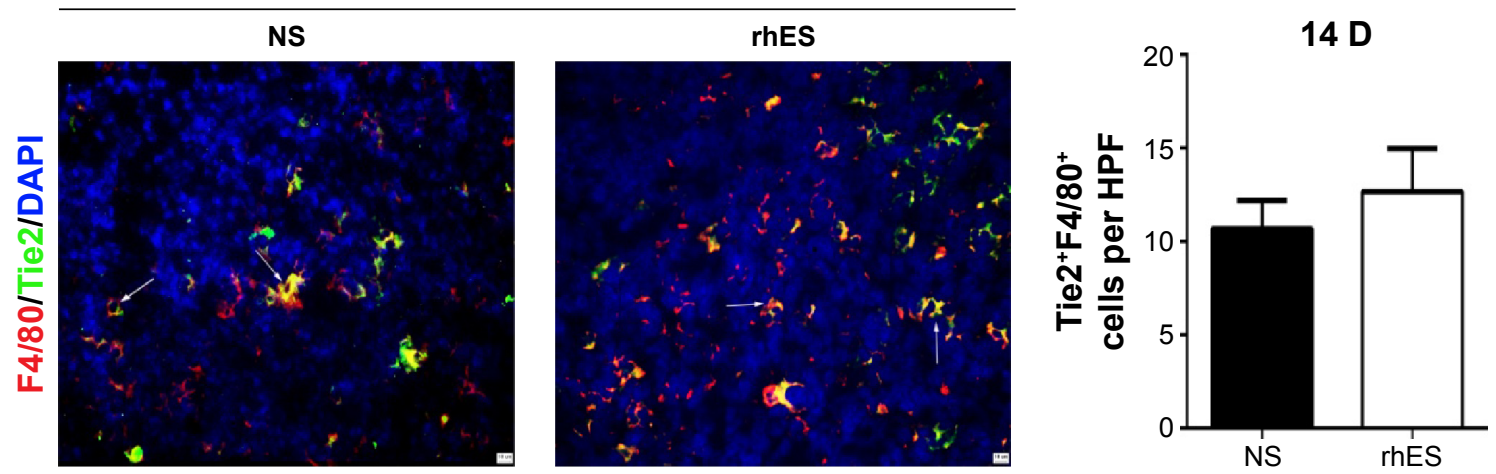

Figure S3 Tie2 ${ }^{+}$macrophages in tumor tissues of LLC-bearing mice treated with recombinant human endostatin (rhES) for I4 days. Representative images of Tie2 stained sections from LLC-bearing mice treated with normal saline (NS) or rhES. The pictures show merged fluorescent Tie2 (green; Santa Cruz Biotechnology Inc., Dallas, TX, USA), F4/80 (red; eBioscience), and DAPI (blue; Sino Biological Inc). White arrows indicate Tie2 ${ }^{+}$cells. LLC-bearing mice were treated with NS or rhES for I4 days. The number of $\mathrm{Tie2}^{+}$macrophages were counted in a high-power field $(\times 200$, scale bar $=10 \mu \mathrm{m})$. Data are presented as mean \pm SD $(n=3$ animals per group).

Abbreviations: LLC, Lewis lung carcinoma; DAPI, 4',6-diamidino-2-phenylindole; SD, standard deviation; D, days. 
OncoTargets and Therapy

\section{Publish your work in this journal}

OncoTargets and Therapy is an international, peer-reviewed, open access journal focusing on the pathological basis of all cancers, potential targets for therapy and treatment protocols employed to improve the management of cancer patients. The journal also focuses on the impact of management programs and new therapeutic agents and protocols on The manuscript management system is completely online and includes a very quick and fair peer-review system, which is all easy to use. Visit http://www.dovepress.com/testimonials.php to read real quotes from published authors.

Submit your manuscript here: http://www.dovepress.com/oncotargets-and-therapy-journal 